\title{
Spontaneous Jamming of Horizontal Semicircular Canal Combined with Canalolithiasis of Contralateral Posterior Semicircular Canal
}

\author{
Salvatore Martellucci ${ }^{1}$, Andrea Castellucci ${ }^{2}$, Pasquale Malara ${ }^{3}$, Giulio Pagliuca ${ }^{1}$, \\ Veronica Clemenzi ${ }^{1,4}$, Andrea Stolfa ${ }^{1,4}$, Andrea Gallo ${ }^{1,4}$, and Giacinto Asprella Libonati ${ }^{5}$ \\ ${ }^{1}$ ENT Unit, Santa Maria Goretti Hospital, AUSL Latina, Italy \\ ${ }^{2}$ ENT Unit, Arcispedale Santa Maria Nuova, AUSL-IRCCS, Reggio Emilia, Italy \\ ${ }^{3}$ Audiology and Vestibology Service, Centromedico Bellinzona, Bellinzona, Switzerland \\ ${ }^{4}$ Department of Sense Organs, Sapienza University of Rome, Italy \\ "U.O.S.D. "Vestibologia e Otorinolaringoiatria" Presidio Ospedaliero "Giovanni Paolo II", Policoro, Italy
}

Received September 22, 2020

Revised November 22, 2020

Accepted December 17, 2020

Address for correspondence

Salvatore Martellucci, $\mathrm{MD}, \mathrm{PhD}$

ENT Unit,

Santa Maria Goretti Hospital,

AUSL Latina, Via GB Lulli 10,

04100 Latina, Italy

Tel $+39-0776551$

Fax $+30-0773902252$

E-mail dott.martellucci@gmail.com
Spontaneous canalith jam is an uncommon form of benign paroxysmal positional vertigo mimicking acute vestibular neuritis. We described for the first time a spontaneous horizontal semicircular canalith jam associated with a typical canalolithiasis involving contralateral posterior semicircular canal (PSC), illustrating how the latter condition modified direction-fixed nystagmus during head movements. An 81-year-old woman with persistent vertigo referred to our center. Video-Frenzel examination showed horizontal direction-fixed right-beating nystagmus in primary gaze position, inhibited by visual fixation. She exhibited corrective saccades after leftward head impulses. Chin-to-chest positioning at the head-pitch test did not modify spontaneous nystagmus, whereas slight torsional components with the top pole of the eye beating toward the right ear appeared in backward head-bending, resulting in mixed horizontal-torsional nystagmus. At supine positioning tests, direction-fixed nystagmus turned into direction-changing geotropic horizontal nystagmus, which was stronger on the left side, while overlapping upbeat nystagmus with torsional right-beating components appeared on the right. Primary clinical findings were consistent with a left horizontal semicircular canalith jam, inducing a persistent utriculofugal cupular displacement, combined with a typical right-sided PSC-canalolithiasis. Once canalith jam crumbled, resulting in a non-ampullary arm canalolithiasis of the horizontal semicircular canal, both involved canals were freed by debris with appropriate repositioning procedures.

J Audiol Otol 2022;26(1):55-60

KEY WORDS: Canalith jam · Benign paroxysmal positional vertigo · Head impulse test Multicanal benign paroxysmal positional vertigo . Bilateral benign paroxysmal positional vertigo.

\section{Introduction}

Benign paroxysmal positional vertigo (BPPV) is considered the most common vestibular disorder. The most reliable pathophysiological mechanism explaining BPPV is otoconial

This is an Open Access article distributed under the terms of the Creative Commons Attribution Non-Commercial License (https://creativecommons.org/licenses/by-nc/4.0/) which permits unrestricted non-commercial use, distribution, and reproduction in any medium, provided the original work is properly cited. displacement from the utricular macula to semicircular canals (SCs). Debris either free-floating in the canal lumen (canalolithiasis) or adhering to the cupola (cupololithiasis) enables the involved canal to become sensitive to gravity, resulting in positional nystagmus and vertigo spells triggered by head positionings. Positional nystagmus is typically transitory and rotates around an axis perpendicular to the plane of the affected SC, which can be identified analysing directions of vertical, horizontal and torsional components in relation to head posi- 
tionings [1].

BPPV can affect one or multiple SCs, either unilaterally or bilaterally. Most cases arise from posterior SC (PSC), less commonly from horizontal SC (HSC), while rarely from anterior SC (ASC) [1]. Although simultaneous involvement of multiple SCs represents an uncommon event, it is not exceptional, accounting for $6-20 \%$ of all BPPV, mainly after head trauma [1,2].

Canalith jam represents an uncommon subtype of BPPV in which canaliths are thought to aggregate plugging the canal lumen. In this condition, entrapped otoliths are assumed to exert either negative or positive pressure on the cupula, preventing dynamic responses of the affected SC by blocking endolymphatic flows, thus resulting in a persistent direction-fixed nystagmus regardless of head positions [3-7]. Canalith jam can occur either spontaneously or as a complication of canalith repositioning maneuvers (CRM) [3-7].

We described a challenging presentation for multicanal $\mathrm{BPPV}$, as the patient herein reported presented with a spontaneous canalith jam involving left HSC combined with simultaneous right PSC-BPPV that modified direction-fixed nystagmus during head positionings. To the best of our knowledge, this is the first report showing a canalith jam associated with a typical canalolithiasis on the contralateral side.

\section{Case Report}

An 81-year-old woman presented with vertigo and nausea arisen upon awaking about five days earlier and exacerbated by head movements. She denied concurrent auditory symptoms. She also excluded recent vestibular symptoms and head trauma.

She exhibited spontaneous right-beating nystagmus, which followed Alexander's law and inhibited by visual fixation. Bedside head impulse test (HIT) on the horizontal plane clearly showed refixation saccades after leftward impulses.

Bedside examination with a left eye-mounted monocular video-Frenzel goggle confirmed purely horizontal right-beating nystagmus with the patient in primary gaze position (Fig. 1A). The "head pitch test" (HPT, also called "bow and lean test") was performed to investigate possible modifications of nystagmus with head position changes. Spontaneous nystagmus was not modified by chin-to-chest positioning (Fig. 1B), while slight torsional right-beating components progressively appeared after $30^{\circ}$-backward head-bending, resulting in mixed horizontal-torsional right-beating nystagmus (Fig. 1C). Torsional components receded once the head returned in primary position. These findings recurred each time the head was bent backward (Supplementary Video 1 in the online-only
Data Supplement). Even though HPT was repeated several times few minutes later, it always showed the same signs. Nystagmus direction did not change even after mild head-shakings in upright position.

Mixed upbeating nystagmus with torsional right-beating components appeared even bringing the patient from the sitting to the supine position ["seated-supine positioning test (SSPT)"] (Fig. 2A). Surprisingly, it changed into long-lasting paroxysmal geotropic horizontal nystagmus by turning the patient's head towards the left side at the "supine head roll test" (SHRT, also called "supine head yaw test") (Fig. 2B). The patient's head was then turned contralaterally, eliciting weaker horizontal paroxysmal geotropic nystagmus compared to the left side. Few seconds later, upbeating paroxysmal nystagmus with torsional right-beating components overlapped and replaced horizontal nystagmus (Fig. 2C) (Supplementary Video 2 in the online-only Data Supplement).

These findings suggested a primary left HSC-canalith jam, which then crumbled transforming into HSC-canalolithiasis, combined with simultaneous right PSC-canalolithiasis.

Left HSC was freed with CRM according to Gufoni [8]. Then, right-sided PSC-BPPV was verified with Dix-Hallpike maneuver and Epley's CRM was performed (Supplementary Video 3 in the online-only Data Supplement). Ten minutes later, positioning tests were repeated, proving that the positional vertigo and nystagmus was disappeared after Gufoni maneuver and Epley maneuver for the left HSC-BPPV canalithiasis and right PSC canalithiasis, respectively. Finally, neither spontaneous/positional nystagmus nor corrective saccades after bedside HIT could be observed when the patient was re-examined two days later, accounting for a complete symptoms receding. The patient expressed her consent to the recording and publication of the videos.

\section{Discussion}

In BPPV, vertigo spells are triggered by head position changes resulting in positional nystagmus. Oculomotor patterns depend on both the affected SC and the position of otoconia in the canal lumen.

Diagnostic approach for BPPV is based on the interpretation of nystagmus according to head positionings. Diagnostic tests in both upright and supine positions include head rotations along the planes of the canals, aiming to achieve the most proper stimulation for two paired SCs. In fact, as each stimulus generating a cupular deflection results in the contraction of coupled ocular muscles, both excitatory and inhibitory stimuli for each SC ampullary receptor result in pathognomonic nystagmus patterns [1]. For example, Dix-Hallpike maneuver on the 
Martellucci S, et al.
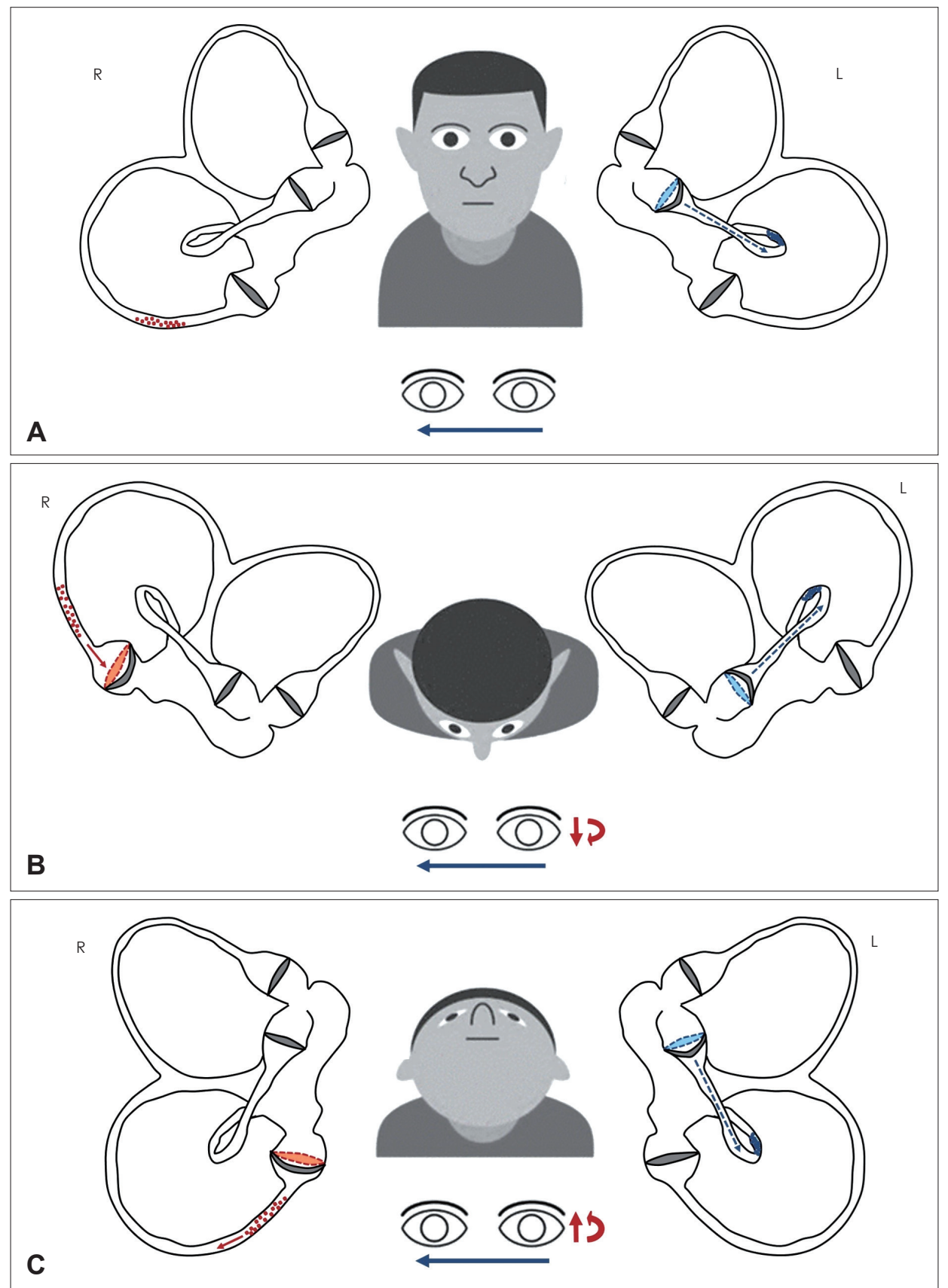

Fig. 1. Schematic representation for the assumed otoliths position within both labyrinths and for resulting nystagmus according to head position changes with the patient upright. Membranous labyrinths (sacculus and cochlea were excluded for simplification) are represented along the sagittal (pitch) plane to show otoliths behavior depending on head movements, thus explaining the proposed mechanism for resulting nystagmus. Debris settling within right and left labyrinths are represented in red and blue, respectively. Nystagmus features (fast phase) resulting from right and left endolymphatic flows are represented with red and blue arrows, respectively. The size of arrows directly correlates with nystagmus amplitude. (A) Purely horizontal spontaneous right-beating nystagmus (blue arrow) in primary gaze position was likely generated by a persistent utriculofugal displacement of left HSC cupula due to a continuous negative pressure (blue dashed arrow) between the otoliths clot (canalith jam) within the HSC and the cupula itself. HSC cupula is shown in utriculofugal (inhibitory) deflection compared to its original position (blue dashed line). Contralaterally, otoliths settle the undermost part of the ampullary arm of right PSC, eliciting neither endolymphatic flows nor detectable nystagmus. (B) Bending the head forward (at the chin-to-chest position), debris were slightly driven toward right PSC cupula (red arrow), resulting in ampullopetal (inhibitory) cupular displacement (red dashed line), generating slight downbeat nystagmus with left-beating torsional components (red arrows). As inhibitory stimuli result in weaker outputs compared to excitatory stimuli, baseline right-beating nystagmus (blue arrow) due to left HSC-canalith jam could have likely overlapped these weak vertical/torsional components. (C) With $30^{\circ}$-backward head-bending at the HPT, particles within right PSC were slightly shifted away from the ampulla (red arrow), resulting in ampullofugal bending of the cupula (red dashed line). This excitatory stimulus likely resulted in an upbeating nystagmus with rightward torsional components (red arrows) overlapping baseline right-beating nystagmus (blue arrow). HSC: horizontal semicircular canal, PSC: posterior semicircular canal, HPT: head pitch test. 

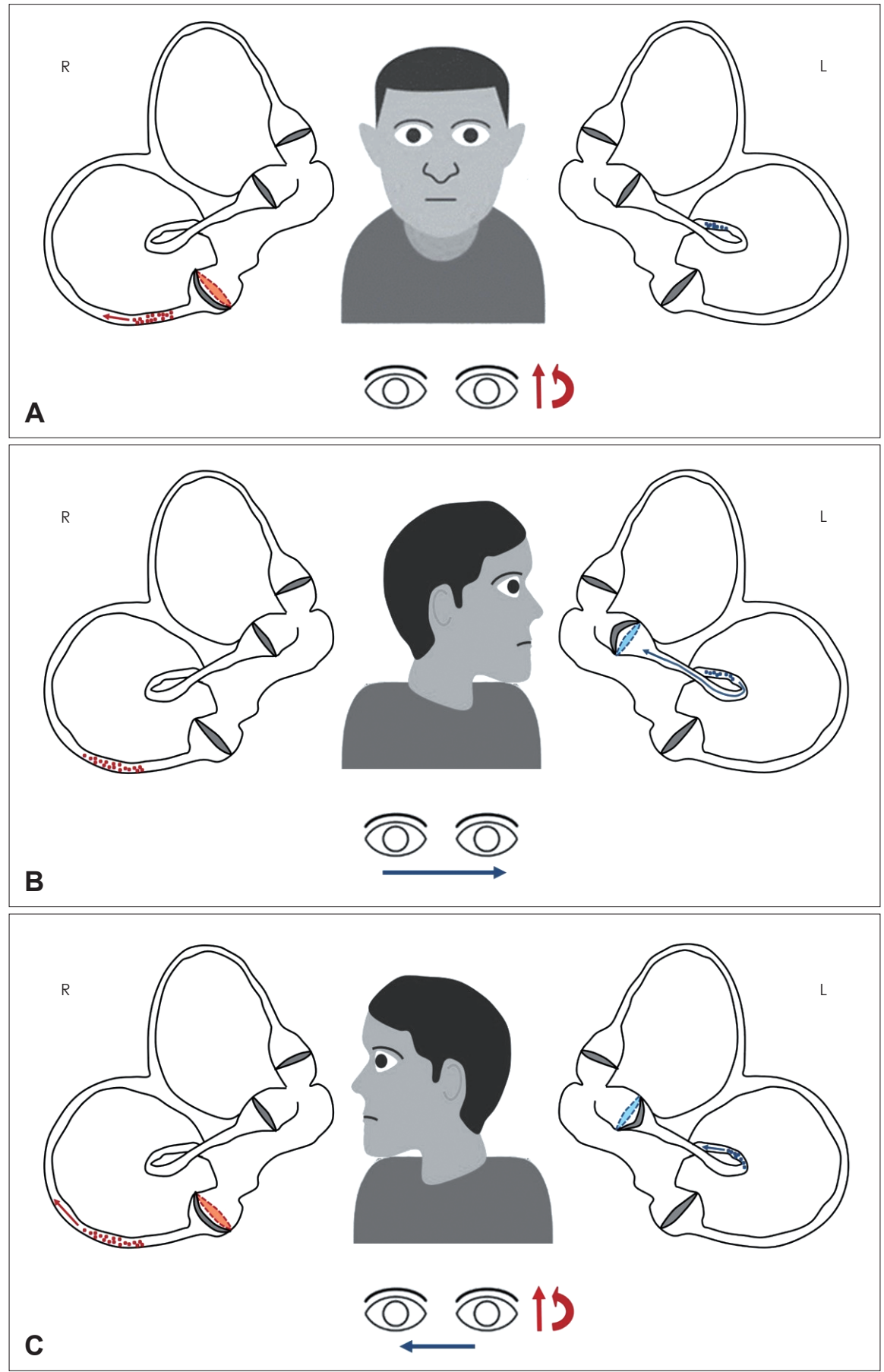

Fig. 2. Representation of particles position within both membranous labyrinths (represented along the pitch plane) and resulting nystagmus according to head position changes with the patient supine. Debris settling within right and left labyrinths are represented in red and blue, respectively. Nystagmus features (fast phase) resulting from right and left endolymphatic flows are represented with red and blue arrows, respectively. (A) At the SSPT, canalith jam likely crumbled within left HSC non-ampullary arm. Contralaterally, debris moved away from the PSC ampulla resulting in an ampullofugal (excitatory) cupular displacement (red dashed line), that in turn generates upbeating nystagmus with torsional right-beating components (red arrows). (B) At leftward SHRT, debris on the left side were displaced toward the HSC ampulla (blue arrow, excitatory stimulus) which in turn bent ampullopetally (blue dashed line) resulting in strong long-lasting paroxysmal geotropic nystagmus (blue arrow). Contralaterally, debris remained in the undermost tract of the PSC without resulting in detectable nystagmus. (C) At rightward SHRT, debris moved away from the ampulla (blue arrow) generating an ampullofugal (inhibitory) endolymphatic flow and weaker right-beating nystagmus compared to contralateral side (blue arrow). On the right side, debris were shifted further away from PSC ampulla (red arrow) resulting in ampullofugal (excitatory) displacement of the ampulla (blue dashed line) and in paroxysmal upbeating nystagmus with right-beating torsional components (red arrows) overlapping and replacing paroxysmal geotropic horizontal nystagmus. SSPT: seated-supine positioning test, HSC: horizontal semicircular canal, PSC: posterior semicircular canal, SHRT: supine head roll test. 
right side mainly assesses both right PSC and left ASC; therefore, positional nystagmus resulting from right PSC-canalolithiasis is upbeating with right-beating torsional components (i.e., with the top poles of the eyes beating toward the lowermost ear) [9].

Although specific positionings have been conceived for the diagnosis of each form of BPPV, positional nystagmus due to a specific BPPV subtype may also be evoked by maneuvers that are recommended for other SCs. Therefore, nystagmus patterns elicited by positionings are more relevant than the positioning itself for BPPV diagnosis [10]. Since BPPV may involve multiple SCs in one or both sides, it may be challenging to identify the position of otoconia and the most appropriate CRM. A nystagmus-guided approach is particularly useful in such cases $[2,10,11]$.

We herein reported a peculiar case of bilateral BPPV including left HSC-canalith jam and right PSC-canalolithiasis.

Canalith jam represents a rare form of HSC-BPPV occurring when an otoliths clot plugs a narrow portion of the membranous duct. In this condition, a continuous alteration of hydrostatic pressure within the affected SC leads to a persistent deflection of the cupula towards the utricle or in the opposite direction. It results in spontaneous nystagmus regardless of head position changes, mimicking vestibular neuritis [3-7].

The patient of our report presented with a history consistent BPPV, showing right-beating horizontal nystagmus in primary gaze position.

Horizontal nystagmus in upright position does not represent a rare finding in HSC-BPPV, as the natural $30^{\circ}$-inclination of HSC from the horizontal plane results in a gravity-induced slow motion of free-floating debris or in a persistent deflection of the overloaded cupula while upright [10-13]. Although it could be misinterpreted as spontaneous nystagmus, it actually represents direction-changing nystagmus as it is strongly modulated by head positions. Therefore, it should be properly called "pseudo-spontaneous nystagmus" due to its gravity dependency [11-14].

However, the patient in this case showed persistent rightbeating nystagmus irrespective to head-bending at HPT and refixation saccades after leftward HIT, consistent with left vestibular neuritis. Nevertheless, it is well known how canalith jam may likely account for these findings in patients with recent BPPV. Persistent utriculofugal displacement of the cupula due to negative pressure between the otoliths clot and the cupula itself, with resulting endolymphatic blockage, may also account for direction-fixed nystagmus and the transient hypofunction of left HSC activity as detected by HIT [2,3,6].

Moreover, upbeating nystagmus with right-beating torsional components regularly overlapped baseline right-beating hori- zontal nystagmus by bending the head $30^{\circ}$ backward. Since head tilts along the pitch plane can stimulate also vertical SCs, such nystagmus pattern indicates the presence of additional debris within the right PSC $[1,10,12]$. This assumption could be verified as similar nystagmus was elicited at the SSPT $[11,13]$. Bringing the patient to the supine position with the head aligned with the body, PSC lies $45^{\circ}$-inclined from the pitch plane and gravity vector can move debris away from PSC cupola resulting in an excitatory stimulus $[10,12,13,15]$. Conversely, slight downbeat nystagmus with torsional left-beating components would have been expected at the chin-to-chest position, due to ampullopetal (inhibitory) otoliths shift within right PSC induced by gravity [12]. Nevertheless, it is well known how inhibitory stimuli for labyrinthine sensors result in weaker oculomotor responses compared to excitatory inputs $[1,9]$. Moreover, slight left-beating torsional components could have been overlapped by stronger baseline right-beating nystagmus. These phenomena may likely account for the lack of nystagmus changes in chin-chest position despite concurrent PSC-canalolithiasis.

Then, SHRT revealed long-lasting, direction-changing paroxysmal geotropic horizontal nystagmus stronger on the left side, suggesting that left HSC-canalith jam crumbled resulting in a settlement of free-floating debris within the non-ampullary arm of the canal [4]. Once the patient's head quickly turned on the right side, torsional components beating toward the undermost ear immediately overlapped horizontal geotropic nystagmus, proving right PSC-canalolithiasis. Right PSCcanalolithiasis was finally verified with Dix-Hallpike test on the right side.

Clinical Practice Guidelines released by American Academy of Otolaryngology-Head and Neck Surgery provide the most appropriate approach in ordinary cases of BPPV, suggesting to check firstly vertical canals involvement with DixHallpike maneuvers, and then HSC-BPPV with SHRT if multicanal involvement is suspected [16]. In this report, however, we described a unique case of left HSC-canalith jam with simultaneous right-sided PSC-canalolithiasis mimicking a clinical picture consistent with Lyndsay-Hemenway syndrome (i.e., positional paroxysmal nystagmus overlapping spontaneous nystagmus and corrective saccades after horizontal head impulse). Once canalith jam crumbled converting into a typical left HSC-canalolithiasis, we followed an unconventional approach based on the regular observation of nystagmus in each head position (nystagmus-based approach).

This kind of approach during positioning tests allowed us to understand canaliths dynamics in both labyrinths and to identify their positions. The same strategy was adopted while performing proper CRM to systematically track the ampullofugal progression of debris within SCs. 


\section{Supplementary Video}

Video 1. Upright examination. Horizontal right-beating nystagmus in primary gaze position. Head pitch test did not modify the direction of spontaneous horizontal nystagmus, whereas slight torsional right-beating components progressively appeared after $30^{\circ}$ backward head-bending.

Video 2. Supine examination. Mixed upbeating nystagmus with torsional right-beating components appeared at the seated-supine positioning test and strong long-lasting paroxysmal geotropic horizontal nystagmus appeared after leftward head rotation. Pure horizontal paroxysmal geotropic nystagmus (weaker than leftward hear rotation) was evoked turning the patient's head to the right side. Few seconds later, up-beating paroxysmal nystagmus with torsional right-beating components overlapped and replaced horizontal nystagmus.

Video 3. Repositioning and final reassessment. No nystagmus appeared in supine position and left supine head roll test, whereas slight upbeating paroxysmal nystagmus with torsional components was still detected on the right.

\section{Supplementary Materials}

The online-only Data Supplement is available with this article at https://doi.org/10.7874/jao.2020.00507.

\section{Acknowledgments}

The authors thank Mr. Livio Fania for the illustrations.

\section{Conflicts of interest}

The authors have no financial conflicts of interest.

\section{Author Contributions}

Conceptualization: Salvatore Martellucci, Andrea Castellucci, and Pasquale Malara. Data curation: Andrea Stolfa. Methodology: Pasquale Malara. Supervision: Andrea Gallo. Writing — original draft: Salvatore Martellucci and Veronica Clemenzi. Writing - review \& editing: Andrea Castellucci, Giulio Pagliuca, Andrea Gallo, and Giacinto Asprella Libonati. Approval of final manuscript: all authors.

\section{ORCID iDs}

Salvatore Martellucci https://orcid.org/0000-0001-5233-2478

Andrea Castellucci https://orcid.org/0000-0003-2874-5672

Pasquale Malara https://orcid.org/0000-0002-7475-9058

Giulio Pagliuca $\quad$ https://orcid.org/0000-0002-2845-4931

Veronica Clemenzi https://orcid.org/0000-0003-1681-4474

Andrea Stolfa https://orcid.org/0000-0001-9221-5575

Andrea Gallo https://orcid.org/0000-0001-8086-4279

Giacinto Asprella Libonati

https://orcid.org/0000-0003-1890-0384

\section{REFERENCES}

1) von Brevern $M$, Bertholon $P$, Brandt $T$, Fife $T$, Imai $T$, Nuti $D$, et al. Benign paroxysmal positional vertigo: diagnostic criteria. J Vestib Res 2015;25:105-17.

2) Shim DB, Song CE, Jung EJ, Ko KM, Park JW, Song MH. Benign paroxysmal positional vertigo with simultaneous involvement of multiple semicircular canals. Korean J Audiol 2014;18:126-30.

3) Schubert MC, Helminski J, Zee DS, Cristiano E, Giannone A, Tortoriello G, et al. Horizontal semicircular canal jam: two new cases and possible mechanisms. Laryngoscope Investig Otolaryngol 2020;5: $163-7$.

4) Castellucci A, Malara P, Brandolini C, Del Vecchio V, Giordano D, Ghidini A, et al. Isolated horizontal canal hypofunction differentiating a canalith jam from an acute peripheral vestibular loss. Am J Otolaryngol 2019;40:319-22.

5) Comacchio F, Poletto E, Mion M. Spontaneous canalith jam and apogeotropic horizontal canal benign paroxysmal positional vertigo: considerations on a particular case mimicking an acute vestibular deficit. Otol Neurotol 2018;39:e843-8.

6) Ko KM, Song MH, Kim JH, Shim DB. Persistent spontaneous nystagmus following a canalith repositioning procedure in horizontal semicircular canal benign paroxysmal positional vertigo. JAMA Otolaryngol Head Neck Surg 2014;140:250-2.

7) Chang YS, Choi J, Chung WH. Persistent direction-fixed nystagmus following canalith repositioning maneuver for horizontal canal BPPV: a case of canalith jam. Clin Exp Otorhinolaryngol 2014;7:138-41.

8) Mandalà M, Pepponi E, Santoro GP, Cambi J, Casani A, Faralli M, et al. Double-blind randomized trial on the efficacy of the Gufoni maneuver for treatment of lateral canal BPPV. Laryngoscope 2013;123: $1782-6$.

9) Eggers SDZ, Bisdorff A, von Brevern M, Zee DS, Kim JS, PerezFernandez N, et al. Classification of vestibular signs and examination techniques: nystagmus and nystagmus-like movements. J Vestib Res 2019;29:57-87.

10) Libonati GA. Benign paroxysmal positional vertigo and positional vertigo variants. Int J Otorhinolaryngol Clin 2012;4:25-40.

11) Asprella-Libonati G. Lateral canal BPPV with pseudo-spontaneous nystagmus masquerading as vestibular neuritis in acute vertigo: a series of 273 cases. J Vestib Res 2014;24:343-9.

12) Choo OS, Kim H, Jang JH, Park HY, Choung YH. Vertical nystagmus in the bow and lean test may indicate hidden posterior semicircular canal benign paroxysmal positional vertigo: hypothesis of the location of otoconia. Sci Rep 2020;10:6514.

13) Yetiser S, Ince D. Diagnostic role of head-bending and lying-down tests in lateral canal benign paroxysmal positional vertigo. Otol Neurotol 2015;36:1231-7.

14) Malara P, Castellucci A, Martellucci S. Upright head roll test: a new contribution for the diagnosis of lateral semicircular canal benign paroxysmal positional vertigo. Audiol Res 2020;10:236.

15) Martellucci S, Attanasio G, Ralli M, Marcelli V, de Vincentiis M, Greco A, et al. Does cervical range of motion affect the outcomes of canalith repositioning procedures for posterior canal benign positional paroxysmal vertigo? Am J Otolaryngol 2019;40:494-8.

16) Bhattacharyya N, Gubbels SP, Schwartz SR, Edlow JA, El-Kashlan $\mathrm{H}$, Fife T, et al. Clinical practice guideline: benign paroxysmal positional vertigo (update). Otolaryngol Head Neck Surg 2017;156(3 suppl):S1-47. 